\title{
RAPID-INTEGRATED NEMOV6 HANDOFF IN IEEE802.16E BWA NETWORKS
}

\author{
Badiea Abdulkarem Mohmmed 1,2 *, TAT-CHEE WAN ${ }^{3}$ \\ ${ }^{1}$ College of Computer Science and Engineering, University of Ha'il, Ha'il, 81481, Saudi Arabia \\ ${ }^{2}$ Faculty of Computer Science and Engineering, Hodeidah University, Hodeidah, Yemen \\ ${ }^{3}$ School of Computer Sciences / National Advanced IPv6 Centre (NAV6), Universiti Sains Malaysia, 11800 \\ USM, Penang, Malaysia
}

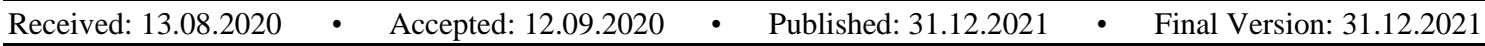

\begin{abstract}
NEMO basic support protocol was created by IETF NEMO working group to extend basic end-host mobility support in, Mobile IP (MIP) protocol to provide network mobility support. However, the handover latency in this scheme is high, and not suitable for multimedia and real time applications. Many techniques have been proposed to solve this problem by parallelizing the dual layers handover and assigning the network layer handover part to the serving Mobile Router (PAR) or to the Home Agent (HA). These techniques either not fully parallelized or its advantages will be lost if the distance between the Mobile Router and its HA is so long. In this paper, we propose a new technique called Rapid-Integrated NEMO Handover (RINEMO) that keep its advantage whatever the distance between the MR and its HA and present a better parallelizing between the link layer and the network layer handover steps. Analytical results comparing the techniques are provided, showing that our technique have the lowest handover latency and lowest disruption time.
\end{abstract}

Keywords: NEMO, IEEE802.16e, Fast -Handover, Mobile IP, WiMAX.

\section{Introduction}

In order to fulfil the demand for on-the-move and uninterrupted internet connectivity in Future Mobile Networks [1,2], the IETF (Internet Engineering Task Force) has standardized NEMO Basic Support Protocol [3,4]. This protocol has been proposed by extending the Mobile IP Protocol [5], to manage the mobility of entire networks instead of managing the mobility of the Mobile Network Nodes (MNNs). In NEMO the Mobile Router (MR) only sends one Binding Update (BU) message to inform its Home Agent (HA) by its Care-of-Address (CoA) that given to it by the visited network. The NEMO basic support protocol provides connectivity to all the mobile nodes within a mobile network Routers, but the handover latency in this protocol is high and not adequate with the multimedia applications like streaming video, VoIP, and music downloads. This high handover latency exists because this protocol focuses on network-layer handover phase and this phase starts after the link-layer handover phase is completed. The current fast handover solutions available in the literature is assigning the process of getting a new CoA to the HA in the home network, wherever, this will cause to loss the advantage of parallelizing the handover steps if the home network is very far. And this will make the previous techniques like FH80216e gives better and more stable results.

The IEEE 802.16 family of standards [6-8] have been developed to meet the demand for Broadband Wireless Access (BWA). The subsequent version of the standard IEEE802.16e [9] have

\footnotetext{
* Corresponding Author: b.alshaibani@uoh.edu.sa
} 
been provided to support the mobile terminals. These standards specify physical (PHY) layer and Medium Access Control (MAC) layer functionalities with many advanced characteristics to provide ubiquitous broad band access and flexible networking for all kinds of terminals. The BWA system provides its own link-layer handover solution for the mobile networks based on IEEE802.16e, but because of the IP addressing and complex mobility pattern in the wireless access network, providing an effective handover scheme to handle all sorts of mobility is still challenge.

The FH80216e [10] was proposed to provide fast and low latency handoff for the Mobile Nodes (MNN) with IEEE802.16e. Lee et. al [11], have proposed an optimization to the link layer handover over the IEEE802.16e. In [17] and [18], Fast-Integrated Handover scheme (FINEMO) was proposed to parallelize the dual layer handoff and gives better results than the FMIPv6 over the IEEE802.16e BWA Networks by assigning the job of getting a new Care-of-Address (CoA) to the home agent (HA) in the home network. Badiea et. al [19], have proposed (MFINEMO) by modifying packets follow during the handover process.

In this paper, a new low latency handover protocol named "Rapid-Integrated NEMOv6 Handover protocol" (RINEMO) is proposed to provide low and stable handover latency in NEMO networks with less disruption time whatever the distance between the Mobile Router (MR) and its Home Agent (HA). This is done by making direct contact between the MR and the New Access Router (NAR) to get a new CoA instead of making the HA the intermediary between them.

The rest of this paper is structured as follows: the brief description of the related work is presented in section 2, section 3 introduces our proposed protocol; the analytical model for evaluating the protocol and the discussion are presented in section 4; and finally, the conclusion and the future work are presented in section 5 .

\section{Related Work}

\subsection{NEMO Basic Support Handover Protocol (BNEMO).}

The BNEMO protocol [4] that provides a conceptual architecture of a mobile network has been developed by the Network Mobility Working Group in the IETF. The basic concept in this protocol is a bi-directional tunnel between the Mobile Router (MR) and its Home Agent (HA). Despite the mobility provided by this protocol, the protocol only focuses on the network layer, and performed its function after the link-layer switch over completion in IEEE802.16 systems. As a result, the handover latency is very high. The handover operation for BNEMO is illustrated in Fig. (1).

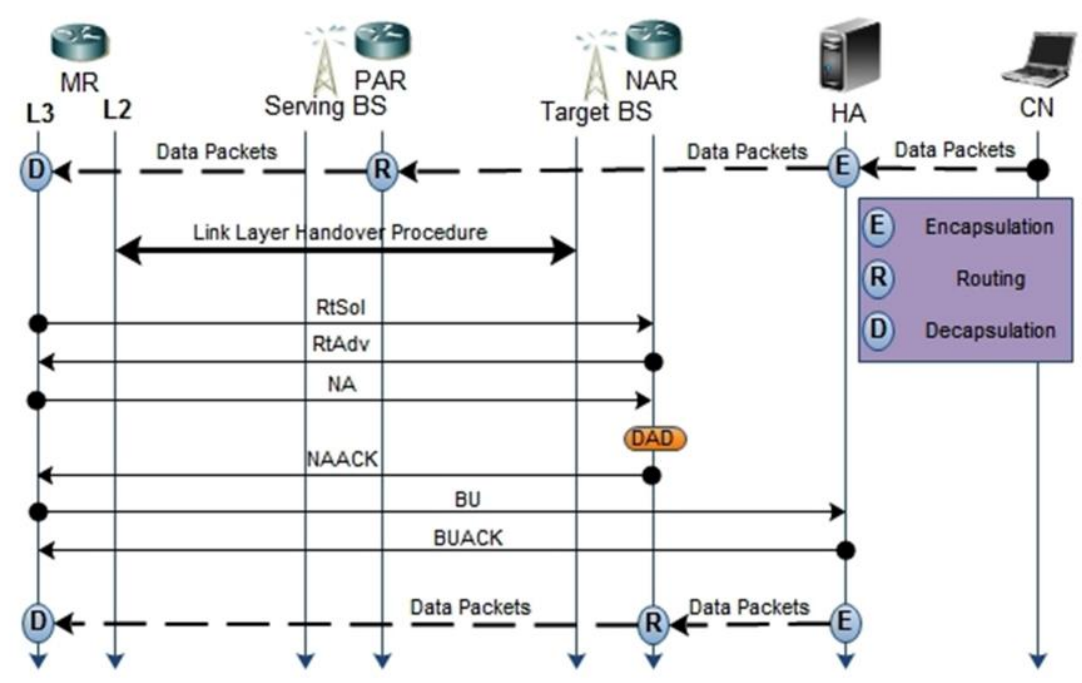

Figure 1. BNEMO Protocol Handover Procedure 
The handover operation in BNEMO is started only after completing the link-layer handover. The Mobile Router (MR) sends a Router-Solicitation (RtSol) immediately. It then receives the router advertisement (RtAdv) message broadcasted by the New Access Router (NAR) and configures its New Care-of-Address (NCoA). After that, MR verifies the uniqueness of the NCoA by sending the Neighbour Advertisement (NA) to the New Access Router (NAR) and subsequently receiving a Neighbour-Advertisement-Acknowledgement (NAACK). As in the Fast Mobile IPv6 Protocol FMIPv6 [5], this step also includes a Duplicate-Address-Detection (DAD) procedure in the NAR. After the NCoA is configured, the MR carries out binding procedure, sending a binding update (BU) to its Home Agent (HA). When HA replies with a binding acknowledgement (BA), its tunnel destination consequently changes from the Previous Care-of-Address (PCoA) to NCoA.

The flow of BNEMO handover is going sequentially starting by the L2 handover steps. The L3 handover steps are started after the L2 handover steps are completed. As a result, high handover latency is produced because the packets will be buffered until the L3 handover process complete.

\subsection{Mobile IPv6 Fast Handovers over IEEE802.16e Networks (FH80216e)}

Jang et. al [10] present FMIPv6 scheme that could be implemented on link layers conforming to the 802.16e specification. This scheme called (FH80216e). In this scheme, two handover modes have been defined: predictive and reactive. In this paper, we consider the predictive mode. They introduced four types of triggers for the interaction between FMIPv6 and IEEE802.16e: New BS Found (NBF), Link Going-Down (LGD), Link Switch (LSW), and Link up (LUP). The predictive mode operation is described in Fig. (3). The handover procedure consists of three phases: neighbour discovery, advance preparation, and handover execution as marked in Fig. (2).

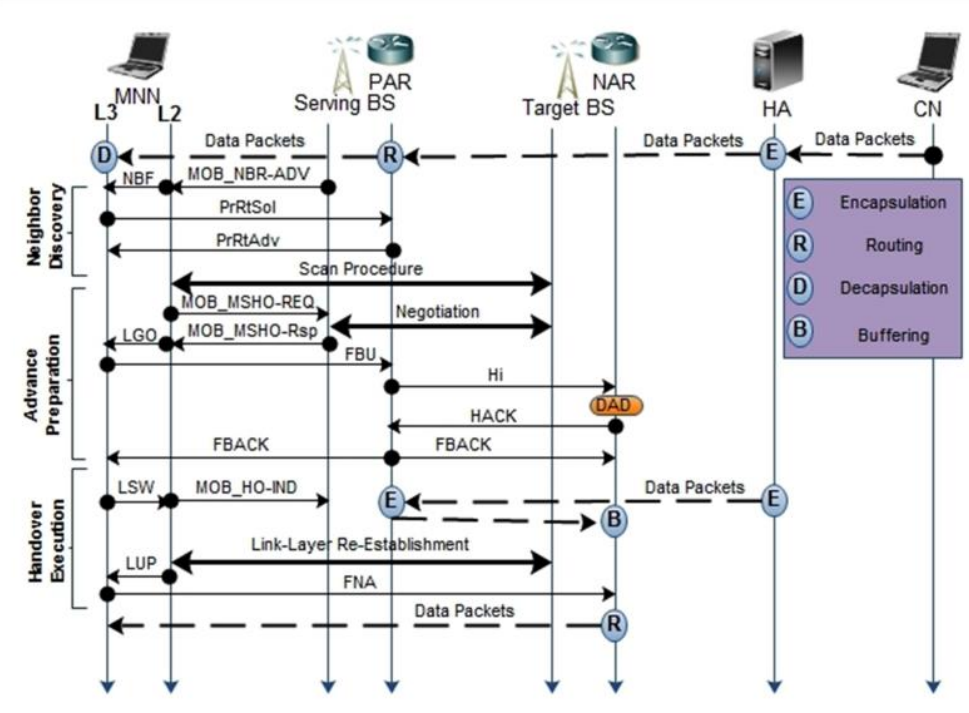

Figure 2. Predictive Mobile IPv6 Fast Handovers over IEEE802.16e

The handover procedure in the predictive mode is briefly described as follows: first, the serving Base Station (BS) broadcasts an IEEE802.16e neighbour advertisement message (MOB_NBR-ADV) periodically. When a new BS is found through the MOB_NBR-ADV and the scanning which maybe performed by MNN, the MNN's link layer (L2) notifies it to the network layer (L3) by NBF trigger. Second, The MNN tries to know more information about the new BS by exchange of Proxy Router solicitation 
(PrRtSol) and Proxy Router Advertisement (PrRtAdv) messages [18] with the Previous Access Router (PAR). Third, The MNN initiates handover by sending an IEEE802.16e handover request (MOB_MSHOREQ) message (MOB means Mobile, MS means Sent by MR, and HO-REQ means Handover Request) to the BS and receives an IEEE802.16e handover response message (MOB_BSHO-RSP) from the BS. When the MNN receives a MOB_BSHO- RSP from the BS, its L2 triggers the L3 by sending the LGD Trigger. On reception of the LGD trigger, the MNN's L3 sends a Fast Binding Update (FBU) message to the PAR. On receiving this message, the PAR establishes tunnel between the PCoA and the NCoA by exchange of $\mathrm{HI}$ and HAck messages with the New Access Router (NAR), and it forwards packets destined for the MNN to the NCoA. Fourth, The MNN receives the FBack message before its handover and sends An IEEE802.16e handover indication (MOB_HO-IND) message as a final indication of handover. Issue of a MOB_HO-IND may be promoted optionally by using a LSW trigger from the L3. Finally, The MNN conducts handover to the target BS and performs the IEEE802.16e network entry procedure. As soon as the network entry procedure is completed, the MNN's L2 signals the L3 with a LUP trigger. On receiving this, L3 issues an Unsolicited Neighbour Advertisement (UNA) message to the NAR by using the NCoA as a source IP address. At the same time, it starts to perform DAD for the NCoA. When the NAR receives the UNA from the MNN, it delivers the buffered packets to the MNN.

The FH80216e protocol defined how to apply the MIPv6 in IEEE802.16e Links. But it did not provide a suitable scheme to parallelize both the L2 and L3 handover steps, and to make this protocol suitable for the NEMO networks, it needs some modification to allow it dealing with the Mobile Routers (MRs) instead of the Mobile Nodes (MNNs).

\subsection{Fast Integrated-Handover Scheme (FINEMO)}

To adapt FH80216e with the NEMO networks and to increase the degree of the parallelization between the L2 and L3 handover steps a modified scheme from FH80216e called Fast IntegratedHandover Scheme (FINEMO) have been proposed in [17] and [18]. The main aim of this scheme is to reduce the Service Interruption time and its negative influence on the real-time traffic. In this

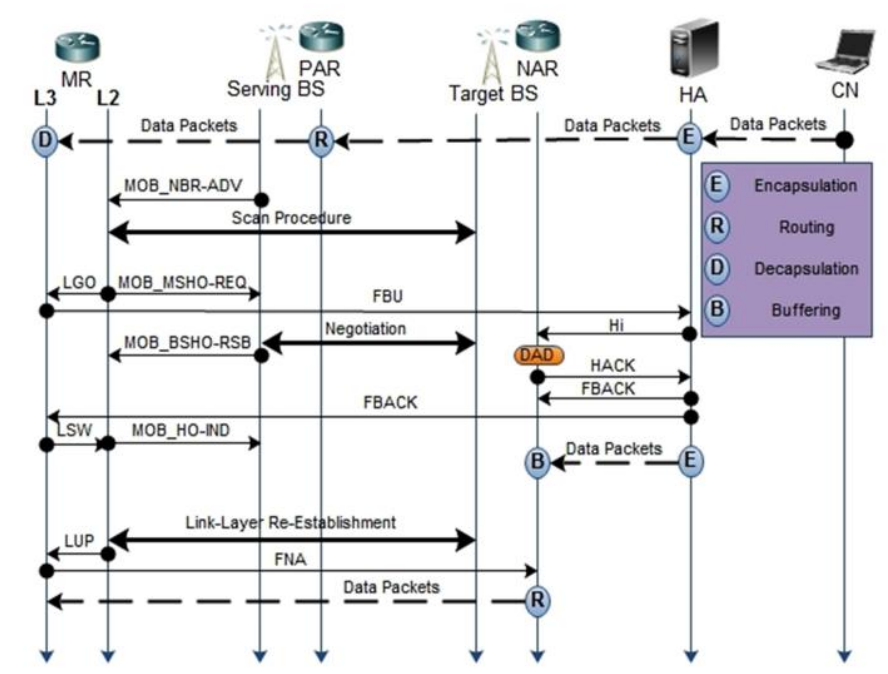

Figure 3. Fast-Integrated Handover Scheme With NEMO Support

scheme, the Neighbour Advertisement (MOB_NBR-ADV) message is being transmitted from time to time by the serving BS. Unlike FH80216e, in this scheme, this message carries both the channel information of the neighbour BS and its Access Router (AR) information. This information would be collected for use during future handovers by the MR. The MR monitors the serving BS radio 
signals. If the signal meets a certain conditions (such as the degrading signal strength or increasing packet loss rate), MR asks for the scanning process to get more information about the selected BS.

At a later time, according to the scanning process information, a concurrent handover will be conducted by the MR. Before the MR disconnects its serving BS, it makes some preparation works called the Advanced Preparation Phase. A concurrent handover has been carried out both in the network and link layers by the MR to save time. Simultaneously, the MR sends a (MOB_MSHOREQ) message to the serving BS and a Fast Binding Update (FBU) message to its HA. The link layer follows the same steps as mentioned in FH80216e, but the network layer follows different ones. When HA receives the FBU, it directly looks for NCoA in the NAR, and the tunnel destination is updated by the HA to the NCoA. After that, the HA replies with a FBack message to MR, which contains a valid NCoA.

Once the MR successfully receives the FBack, it sends an IEEE802.16e (MOB_HO-IND) message to start the handover execution phase. Upon receiving the FBack, the MR causes the LSW to manage handover by sending a MOB_HO-IND message to the target BS and then carries out 802.16e network re-entry process. Once it is completed, the MR's link layer signals its network layer with a (LUP) trigger to send an Unsolicited Neighbour Advertisement (UNA) message. The NAR in its turn sends the buffered packets to MR. This scheme is illustrated in Fig. (3).

This scheme has been succeed to provide a way to make both the L2 and L3 handover steps working in parallel by assigning the L3 steps to the MR's HA, but it looses its advantage when the MR is very far from its HA. Also this scheme has not considered the nested networks and the pinball problem.

\subsection{Modified FINEMO (MFINEMO)}

To overcome the lack of the nested NEMO networks with a sufficient fast handover protocol, Badiea et. al [19] proposed a Modified Fast-Handover scheme (MFINEMO) working in the Light-NEMO

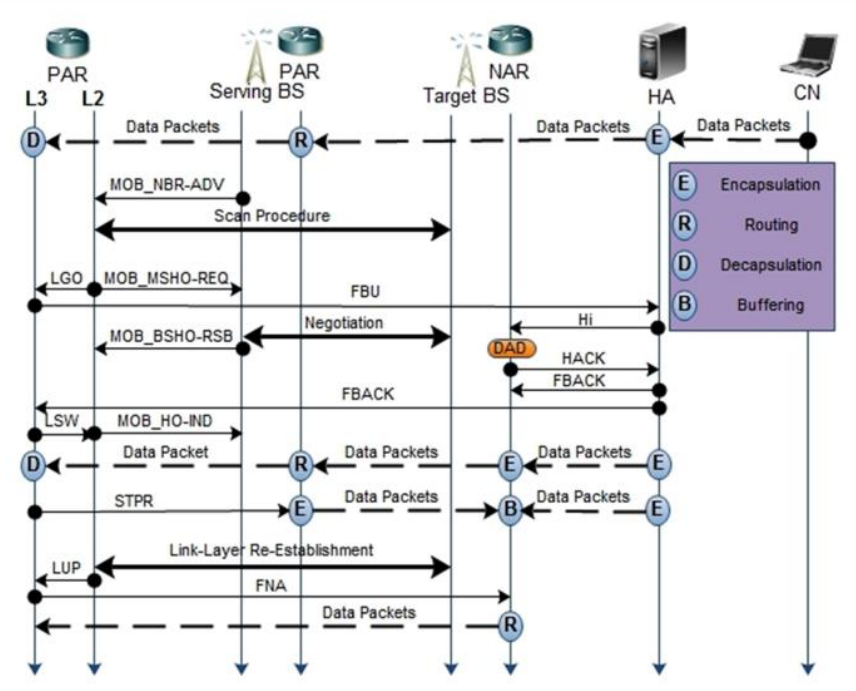

Figure 4. Modified Fast-Integrated Handover Scheme With NEMO Support

network model [11-16]. This scheme has combined the advantages of the FINEMO handover protocol and Light-NEMO network model. In this scheme, a modification to the FINEMO has been proposed too. 
The difference between FINEMO and MFINEMO is in the packet forwarding after assigning the new COA to the MR. In FINEMO the packets will be buffered in NAR until the L2 handover operation has finished and MR has been completely connected to NAR. In the meantime, the NAR with MFINEMO will tunnel the packets to the PAR. PAR in his side will continue sending the packets to the target MR until it receives the Stop Routing (STPR) message from MR. The packets in PAR which have not received by MR yet will be tunnelled back to NAR. When the packets sent by PAR received by NAR, it will then be buffered and all the packets coming from the HA will buffered too until MR is completely handedoff to NAR. This modification managed to reduce significantly the service disruption time, leading to less packet loss. The MFINEMO procedure is illustrated in Fig. (4).

This scheme also did not success to solve the lake of the FINEMO scheme when the MR is so far from its HA. The handover latency in this scheme is high when the distance between the MR and its HA is longer then 17 hops.

\section{PROPOSED WORK}

A proposed fast NEMO handover scheme named Rapid Integrated NEMO (RINEMO) handover scheme is proposed in this paper. This scheme is compatible with FINEMO for network mobility based on the NEMO network model architecture. The basic idea in RINEMO is to apply new prediction mechanisms to optimize NEMO basic support handover procedure. To reduce the handover latency whatever the distance between The MR and its HA, In this proposed scheme, a concurrent process is conducted in both the link layer (L2) and the network layer (L3). Fig. (5) shows the operation of the RILNEMO scheme. The handover procedure consists of three phases: neighbour

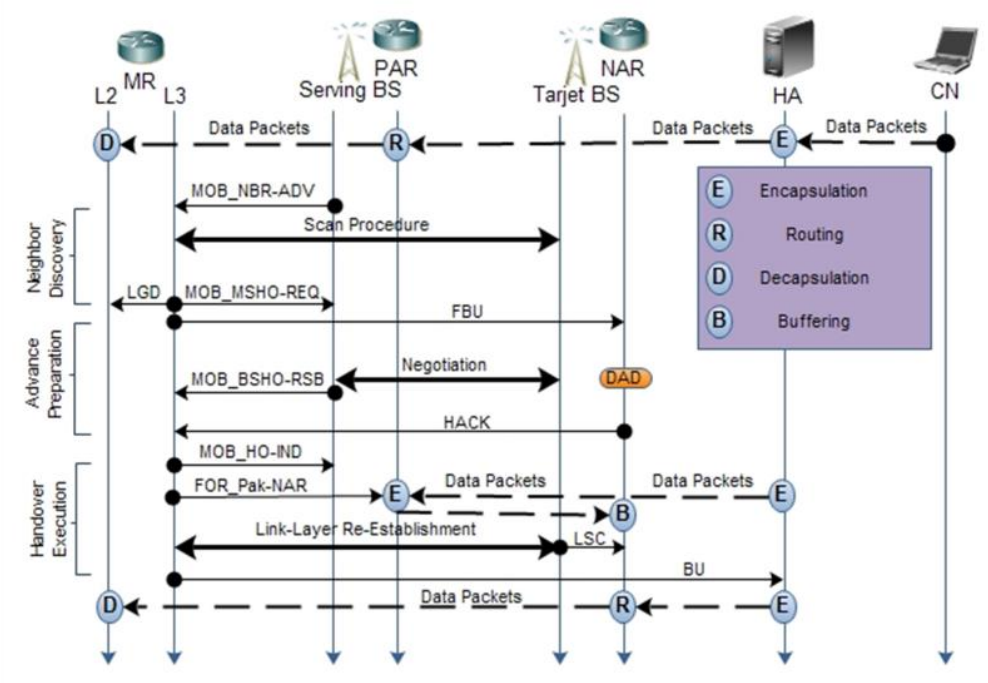

Figure 5. Proposed Rapid-Integrated Light-NEMOv6 Handover

discovery, advance preparation, and handover execution. Two types of triggers are introduced for the interaction between the network layer and the link layer: Link Going-Down (LGD) and Link Switch Complete (LSC). The three phases of this proposed scheme will be explained as follows:

\subsection{Neighbour Discovery}


In this phase, the serving BS periodically sends the link-layer neighbour advertisement message, MOB_NBR-ADV to the broadcast address. However, in our scheme, this message carries not only the channel information of the neighbour BSs but also its AR information, such as the number of neighbouring BS, their BSIDs, their corresponding AR IP addresses, etc. The MR collects this information for future handovers. At the same time, MR monitors the serving BS radio signal strength in every physical-layer frame. If it satisfies a certain condition (such as the degrading signal strength or increasing packet loss rate) the MR requests the scanning process to get more updated and exact information about the selected neighbour BS.

\subsection{Advance Preparation}

Afterwards, depending on the scanning result, the MR may trigger a handover by a pre-defined threshold. However, before it disconnects its serving BS, it does some preparation work, namely, the advance preparation phase. The MR carries out a concurrent handover procedure both in the network and link layers to overlay the sequences of the two layers and save time. It sends a MOB_MSHOREQ message to the serving BS and an FBU to the New Access Router (NAR) simultaneously. The serving BS will start the negotiation process with the new BS and then responds by the MOB_BSHORSB Message. At the same time, NAR will apply the DAD Algorithm to assign a new CoA to the MR. Then, response with the HACK message. The advantages of this operation that the MR will deal directly with the NAR and this will eliminate any extra encapsulations by the PAR or the HA. Second advantage, the distance between the MR and its HA will not affect the process time. The other Advantage is that the data packets will still forwarding to MR with out any disruption time

during this phase.

\subsection{Handover Execution}

This phase will start when the MR sends a MOB_HO-IND message to its serving BS and at the same time sends a new proposed message called FOR_Pack-NAR to inform the PAR that all the receiving packets have to be forwarded to its new CoA. The NAR in its side will buffer these packets until the MR is completely connected with its new BS. After the IEEE802.16e network re-entry process, a LSC trigger is sent from the target BS to the NAR to inform sending the FBACK message to the MR

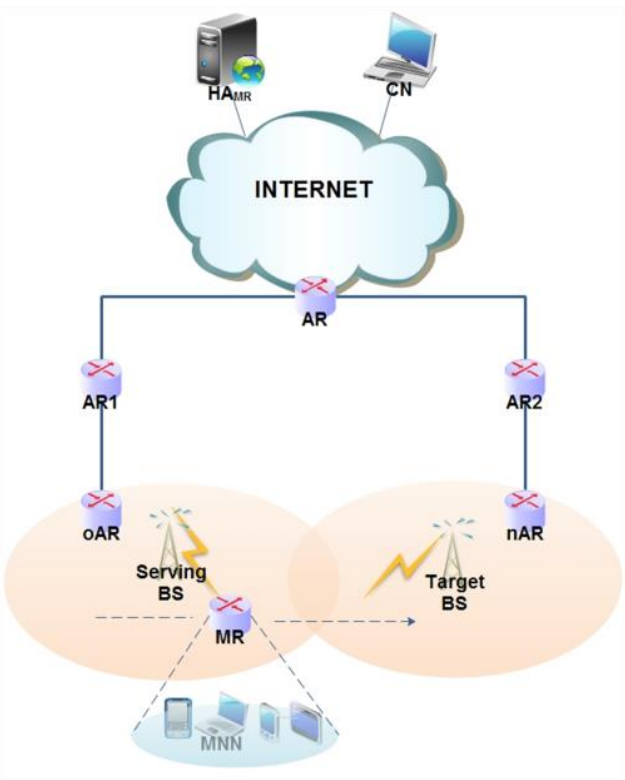

Figure 6. The Network Model 
and starting to send the buffered packets to the MR. the MR then sends the BU message to its HA and receives the data packet resulting from successfully receives the FBACK message.

\section{ANALYSIS AND DISCUSSION}

\subsection{The Network Model}

As showed in Fig. (6), the MR in our network model is going to handoff from the oAR IEEE802.16e link to the nAR IEEE802.16e link, and during that it has a connection with the Corresponding Node $(\mathrm{CN})$. From this model we can notice that the distance between oAR and MR's HA, and the distance between nAR and MR's HA is equivalent. In our mathematical model we will refer to oAR by the Previous Access Router (PAR), and to nAR by the New Access Router (NAR).

\subsection{Analytical Models}

To evaluate and highlight the improvements in our proposed scheme an analytical model based on the analytical model in [16] and [17] will be performed. In this analytical model we have these assumptions:

1. The IEEE802.16e air interface is based on the OFDMA frames.

2. The message processing time $(<1 m s)$ is less than the frame duration.

3. The radio propagation delay $\left(10^{-2} \mathrm{~ms}\right)$ can be omitted.

The definition of the identifiers in the equations is as follows:
1. $t$ frame
Frame duration of IEEE802.16e OFDMA PHY.
2. $t_{b s_{-} \text {nego }}$
Negotiation delay between the serving BS and the
3. ${ }^{t}$ L2_entry recommended BS.
4. ${ }^{\text {hope }}$
Delay of every routing hop in wired backbone network.
5. $d_{N A R_{-} H A}$ Latency of IEEE802.16e network re-entry
procedure.
6. $d_{P A R_{-} H A}$
7. $d_{P A R_{-} N A R}$
Distance between the NAR and the HA in hops.
8. ${ }^{t} D A D$
Distance between the PAR and the $H A$ in hops.
9. ${ }^{t} \mathrm{CN}_{-} \mathrm{HA}$
Distance between the $P A R$ and the NAR in hops.
10. $[X]_{\text {frame }}$

$$
\begin{aligned}
& \text { Time needed to perform a } D A D \text { process. } \\
& \text { Link delay between } C N \text { and } H A \text {. } \\
& \text { Rounding the element of } X \text { to the nearest integer }
\end{aligned}
$$ toward infinity.

\subsection{Handover Latency Analysis}

The definition of the Handover latency here is the interval between the start of the handover process and the time the MN can send and receive data packets in the visited network. In our proposed RINEMO, the handover process starts when the MR sends MOB_MSHO-REQ message until the MR decides to send BU message. Due to the parallelizing of L2 and L3 handover steps, the total handover time will be the maximum time between them. The negotiation time between the serving BS and the target BS $\left(t_{b s_{-} n e g o}\right)$ is related with routing hop delay $\left(t_{h o p}\right)$, and the latency of the 
IEEE802.16e re-entry procedure $\left(t_{L 2 \_ \text {entry }}\right)$ is related with the frame duration in IEEE802.16e $\left(t_{\text {frame }}\right.$

). These relations given by the following equations:

$T_{L_{2} \text { entry }}=10 t_{\text {frame }}+20(\mathrm{~ms})$

$t_{B S_{-} N e g o}=2\left(\left(d_{P A R_{-} N A R}\right) \times t_{h o p}+2 t_{h o p}\right)$

The total handover time for our RILNEMO protocol can be expressed as follows:

$$
\begin{gathered}
T_{H O}^{\text {RINEMO }}=\operatorname{Max}\left(T_{L_{2} \text { Prepare }}^{\text {RINEMO }}+\left(T_{L_{2} \text { entry }}+t_{\text {frame }}\right),\right. \\
\left.T_{L_{3-} \text { Prepare }}^{\text {RINEMO }}\right)+t_{B U}^{\text {RINEMO }}
\end{gathered}
$$

Where is

$T_{L_{2-} \text { Prepare }}^{\text {RINEMO }}=4 t_{\text {frame }}+\left[t_{B S_{-} \text {Nego }}\right]_{\text {frame }}$

$T_{L_{3} \text { Prepare }}^{\text {RINEMO }}=3 t_{\text {frame }}+\left[2 t_{\text {hop }}\left(d_{\text {PAR_NAR }}+1\right)+t_{\text {DAD }}\right]_{\text {frame }}$

And

$t_{B U}^{R I N E M O}=t_{\text {frame }}+\left[2 t_{\text {hop }}\left(d_{\text {NAR_HA }}+1\right)\right]_{\text {frame }}$

FINEMO and BNEMO Handover procedures can be expressed by the following equations:

$T_{H O}^{\text {FINEMO }}=\operatorname{Max}\left(T_{L_{2} \text { Prepare }}^{\text {FINEMO }}+T_{L_{2 \_} \text {entry }}, T_{L_{3}-\text { Prepare }}^{\text {FINEMO }}\right)+t_{\text {FBack }}^{\text {FINEMO }}$

Where is

$T_{L_{2}-\text { Prepare }}^{\text {FINEO }}=4 t_{\text {frame }}+\left[t_{B S \_ \text {Nego }}\right]_{\text {frame }}$

$T_{L_{3}-\text { Prepare }}^{\text {FINEO }}=3 t_{\text {frame }}+\left[2 t_{\text {hop }}\left(d_{\text {PAR_HA }_{-} H}+d_{N_{\text {NAR_HA }} H}+1\right)+t_{\text {DAD }}\right]_{\text {frame }}$

And

$t_{\text {FBack }}^{\text {FINEMO }}=t_{\text {frame }}+\left[2 t_{\text {hop }}\right]_{\text {frame }}$

$T_{H O}^{B N E M O}=T_{L_{2} \text { Prepare }}^{\text {BNEMO }}+T_{L_{2} \_ \text {entry }}+T_{\text {CoA }}^{B N E M O}+t_{B U}^{B N E M O}$

Where is

$T_{L_{2}-\text { Prepare }}^{B N E M O}=4 t_{\text {frame }}+\left[t_{B S \_ \text {Nego }}\right]_{\text {frame }}$

$T_{\text {CoA }}^{B N E M O}=6 t_{\text {frame }}+\left[2 t_{\text {hop }}\right]_{\text {frame }}+\left[2 t_{\text {hop }}+t_{\text {DAD }}\right]_{\text {frame }}$

And

$t_{B U}^{B N E M O}=3 t_{\text {frame }}+\left[2 t_{\text {hop }}\left(1+d_{N_{\text {NAR_HA }} H}\right)\right]_{\text {frame }}$ 


\subsection{Service Disruption Time Analysis}

The service disruption time is the period when the MR cannot send or receive data packets. In other words, it is the time between disconnecting from the serving BS and connecting to target BS. In our RINEMO, the MR cannot receive data packets when it sends FOR_PACK-NAR message to the PAR until it finished performing the re-entry procedure. However, the MR will be able to receive data packets from the time the new BS sends the LSC trigger to the NAR and even before sending the BU message. The service disruption times for our RINEMO and the other schemes are represented by the following equations:

$$
\begin{aligned}
& S D^{\text {RINEMO }}=t_{L_{2} \text { entry }} \\
& S D^{\text {FINEMO }}=t_{L_{2} \text { entry }}+t_{\text {FBack }} \\
& S D^{\text {BNEMO }}=t_{L 2 \_ \text {entry }}+t_{C O A}+t_{B U}
\end{aligned}
$$

\subsection{Packet Loss Analysis}

The packets is queued in the limited size NAR and PAR buffers during the service disruption time, so reducing the service disruption time will improve the QoS and reduce the packet loss. In RINEMO, we have made the service disruption time less than other schemes and that makes our RILNEMO more tolerance to the packet loss challenge.

\subsection{Packet Delay analysis}

We mean by the packet delay here is the time needed to deliver the first packet from $\mathrm{CN}$ to MR over the new link after finishing the handover process. Usually, the packet must be already sent and buffered in NAR queue. The time delay here will be the time consumed to send the packet from the $\mathrm{CN}$ to the HA, plus the time needed to deliver the packet from the HA to the NAR, plus the packet waiting time in the NAR (W), and plus the time needed to send the packet from NAR and received it by the M. The enhancement provided by our RINEMO in packet delay issue is done by making the waiting time in the NAR queue is less than any other scheme. This can be expressed as follows:

$$
\begin{aligned}
& P D^{R I N E M O}=t_{\text {frame }}+\left(d_{P A R_{-} H A}+d_{P A R_{-} N A R}+1\right) \times t_{\text {hop }}+W^{\text {RINEMO }}+t_{C N_{-} H A} \\
& P D^{\text {FINEMO }}=t_{\text {frame }}+\left(d_{N_{\text {AR_HA }} H}+1\right) \times t_{\text {hop }}+W^{\text {FINEMO }}+t_{C N_{-} H A} \\
& P D^{B N E M O}=t_{\text {frame }}+\left(d_{N A R_{-} H A}+1\right) \times t_{h o p}+W^{B N E M O}+t_{C N_{-} H A} \\
& W^{\text {RINEMO }}=t_{L_{2} \text { Entry }}-\left(d_{\text {NAR_HA }} \times t_{\text {hop }}\right) \\
& W^{\text {FINEMO }}=t_{L_{2} \text { Entry }}+t_{\text {FBack }} \\
& W^{B N E M O}=t_{L_{2} \text { Entry }}+t_{C O A}+t_{B U}
\end{aligned}
$$

\subsection{Numerical Results}


The parameters values that have been used in our numerical analysis to produce our numerical results are stated in Table (1) and the final results of our numerical analysis are shown in Fig. (8).

Table 1. Some Parameters Assumptions.

\begin{tabular}{|l|c|}
\hline$d_{P A R_{-} N A R}$ & 4 hops \\
\hline${ }^{t}$ hop & $0.5 \mathrm{~ms}$ \\
\hline${ }^{t}$ DAD & $\begin{array}{c}800 \\
\mathrm{~ms}\end{array}$ \\
\hline
\end{tabular}

Fig. [7(a)] shows the relation between the handover latency and the frame duration and it can be seen that our proposed RINEMO has the lowest handover latency. In this result we have assumed that the distance between NAR (or PAR) and the MR's HA is 20 hops. In Fig. [7(b)], the frame duration has been assumed to be constant equal to $5 \mathrm{~ms}$, and the distance between MR and its HA is our variable. This figure shows that the proposed RINEMO has the lowest handover latency whatever the distance between MR and its HA, since the HA does not take any part of the work. In current practical scenarios, it is more common that the MR is apart from its HA of less than 12 hops within an operator's network [15].

The same parameters were used in the handover latency evaluation have been used for the service disruption time evaluation. Fig. [7(c)] and Fig. [7(d)] show the service disruption time in our RINEMO and the other schemes. It is clear that in our proposed RINEMO the service disruption time is lower than FINEMO and BNEMO.

By assuming that the $t_{\text {frame }}$ is 5 we can see that the packet delay situation between $\mathrm{CN}$ and MR is more enhanced in RILNEMO as shown in Fig. [7(e)]. Due to the decreased of the waiting time of the packet in the NAR queue in our proposed RINEMO, the packet delay in the proposed RINEMO is lower than FINEMO and BNEMO. As a result, our RILNEMO gives better enhancement in the terms of quality of service in the Mobile Networks.

\section{CONCLUSIONS}

The RINEMO that proposed in this paper which supports NEMO in IEEE802.16e networks has the advantages of parallelizing the L2 and the L3 handover procedure steps without affected by the distance of the MR and its HA and provides the most fast known integrated handover scheme. As a result of that, the proposed RINEMO which have better handover latency, lowest service disruption time and better QoS compared to existing schemes, is more adequate to be used in the mobile networks to provide more QoS for the multimedia and the real time applications.

In future work, a simulation which evaluates the new scheme is planned to be presented. Also, we plan to further optimize the individual steps in the handover procedure. The routing optimization will also be investigated and enhanced to provide a better QoS performance.

\section{ACKNOWLEDGMENT}

The authors would like to thank Universiti Sains Malaysia for its financial support. 


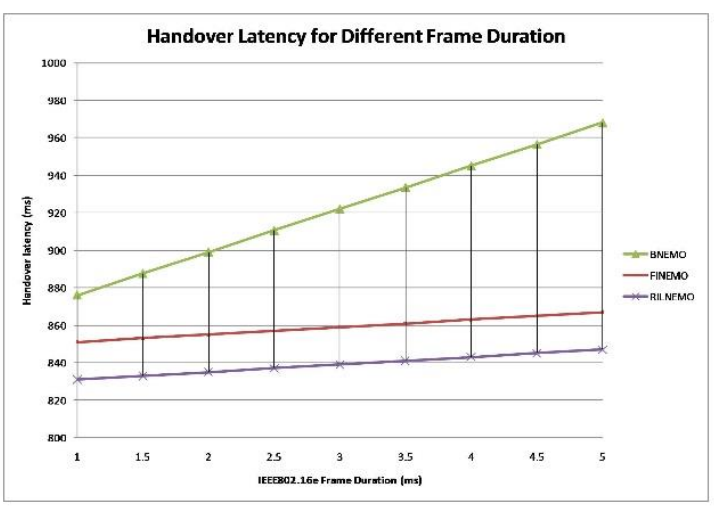

Fig. 7(a). Handover Latency for Different Frame Duration

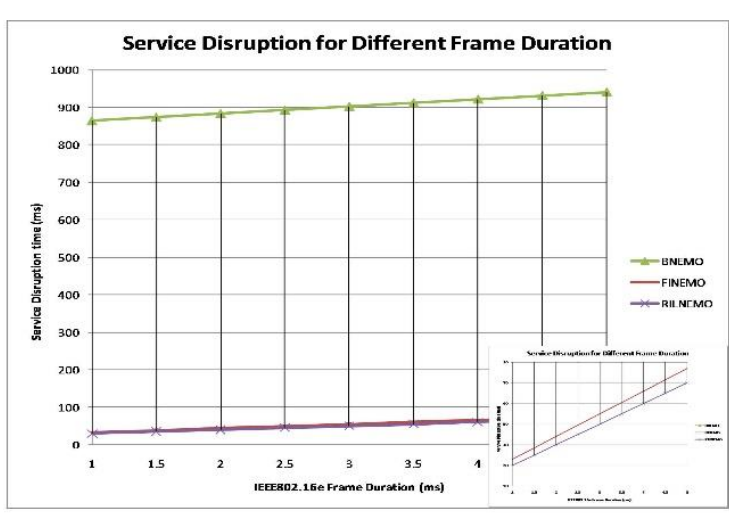

Fig. 7(c). Service Disruption Time for Different Frame Duration

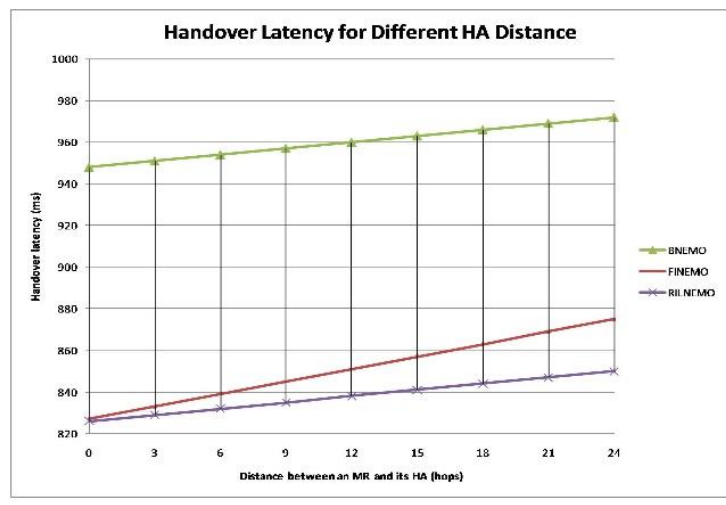

Fig. 7(b). Handover Latency for Different Distance between MR and HA

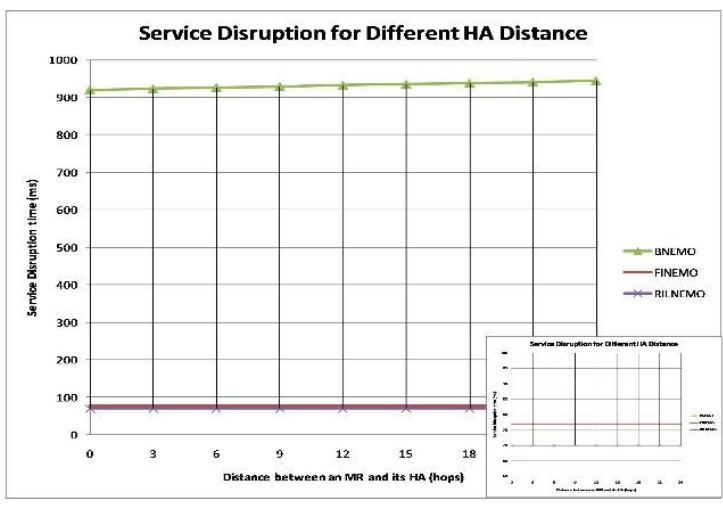

Fig. 7(d). Service Disruption Time for Different Distance between MR and its HA

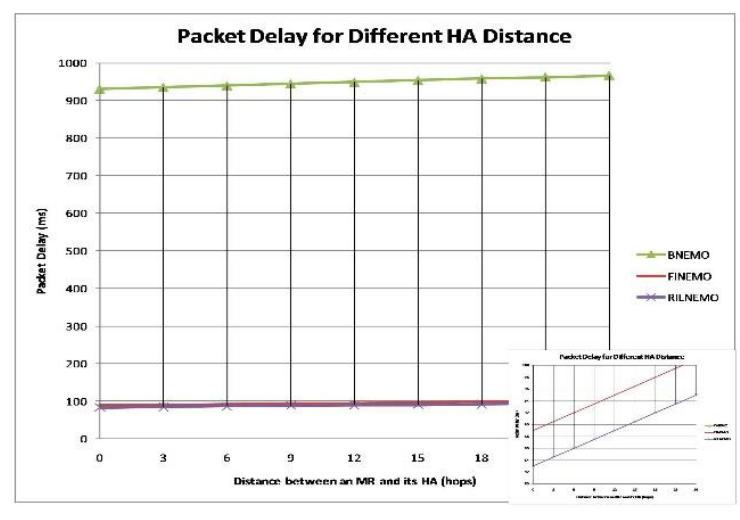

Fig. 7(e). Packet Delay for Different Distance between MR and its HA

Figure 7. Various Handover Latency, Service Disruption Time, and Packet Delay Numerical Results 


\section{REFERENCES}

1. Suk, Y. H.; and Kai, H. Y., "Challenges in the migration to 4G mobile systems", Journal of Communications Magazine, IEEE, Vol. 41, No. 12, 2003, PP 54-59.

2. Angjo, J., et al. "Handover Management of Drones in Future Mobile Networks: 6G Technologies", IEEE Access, Vol 9, 2021, pp 12803-12823.

3. Devarapalli, V.; Wakikawa, R.; Pertruscu, A.; and Thubert, P., "Network mobility (NEMO) basic support protocol", IETF RFC 3963, 2005.

4. David, A. O., and Osofisan, A., "Route Optimization in MIPv6 Experimental Test bed for Network Mobility: Tradeoff Analysis and Evaluation", International Journal of Computer Science and Information Security (IJCSIS), Vol. 18, No. 5, 2020, pp. 19-28.

5. Johnson, D.; Perkins, C.; and Arkko, J., "Mobility Support in IPv6", IETF RFC 377, 2004.

6. Eklund, C.; Marks, R.B.; Stanwood, K.L.; and Wang, S., "IEEE Standard 802.16: a Technical Overview of the WirelessMAN ${ }^{\mathrm{m}}$ Air Interface for Broadband Wireless Access", Communications Magazine, IEEE, Vol. 40, No. 6, 2002, pp 89-107.

7. Ghosh, A.; Wolter, D.R.; Andrews, J.G.; and Chen, R., "Broadband wireless access with WiMax/802.16: current performance benchmarks and future potential", Communications Magazine, IEEE, Vol. 43, No. 2, 2005, pp 129-136.

8. IEEE, "IEEE Standard for Local and metropolitan area networks Part 16: Air Interface for Broadband Wireless Access Systems", IEEE Std 802.16-2009 (Revision of IEEE Std 802.16-2004), 2009.

9. IEEE, "IEEE802.16e Standard-Local and Metropolitan Area Network-Part 16: Air Interface for Fixed and Mobile Broadband Wireless Access Systems", 2005.

10. Jang, H.; Jee, J.; Han, Y. H.; Park, S. D.; and Cha, J., "Mobile IPv6 fast handovers over IEEE802.16e Networks", IETF RFC 5270, 2008.

11. Lee, D. H.; Kyamakya, K.; and Umondi, J. P, "Fast Handover Algorithm for IEEE802.16e Broadband Wireless Access System", Proceeding of $1^{\text {st }}$ International Symposium on Wireless Pervasive Computing, Phuket, Thailand, 2006, pp 16-18.

12. Sabeur, M.; Jouaber B.; and Zeghlache, D., "Fast Light-NEMO: A Seamless Handoff Solution for Nested Mobile Networks", The proceeding of the International Conference on Wireless Networks (ICWN07), Las Vegas, Nevada, USA, 2007, pp 251-257.

13. Sabeur, M.; Jouaber, B.; and Zeghlache, D., "MR-Proxy based solution for nested Mobile Network Problems", Proceeding of Wireless Personal Multimedia Communications (WPMC 2005), Aalborg, Denmark, 2005, pp 21-28.

14. Sabeur, M.; Jouaber, B.; and Zeghlache, D., "Seamless Handoff Solution For Nested Mobile Networks". Journal of Networks (JNW), Vol. 1, No. 4, 2006, pp 21-28.

15. Sabeur, M.; Jouaber, B.; and Zeghlache, D., "Low Latency Handoff for Nested Mobile Networks", Proceeding of IEEE Consumer Communications and Networking Conference (CCNC 2006), Las Vegas, USA, 2006, Vol. 1, pp 492-496.

16. Sabeur, M.; Jouaber, B.; and Zeghlache, D., "Light-NEMO+: Route Optimization for Light-NEMO Solution", Proceeding of the $14^{\text {th }}$ IEEE International Conference on Networks (ICON 2006), Singapore, 2006, Vol. 2, pp 528-533.

17. Zhong, L.; Liu, F.; and Yusheng J., "Fast-integrated handover scheme with NEMO support in IEEE802.16e BWA networks", Proceeding of APAN Network Research Workshop, Xi'An, China, 2007, pp 85-90.

18. Zhong, L.; Liu, F.; and Yusheng J., "Fast Handover Scheme for Supporting Network Mobility in IEEE802.16e BWA System", Proceeding of International Conference on Wireless Communications, Networking and Mobile Computing ( WiCom 2007), Shanghai, China, 2007, pp 85-90.

19. Mohammed, B. A.; and Wan, T. C., "Modified Fast-integrated Light-NEMOv6 handoff in IEEE802.16e BWA Networks", Proceeding of the Second International Conference on Network Applications, Protocols and Services (NETAPPS 2010), Alore setar, Kedah, Malaysia, 2010, pp 182-187.

20. Koodli, R. Ed., "Mobile IPv6 Fast Handovers", IETF RFC 5268, 2008.

21. Kim, Y. B.; Park, Y. J.; Kim, S; and Huh, E. N., "Route Optimization Problems with Local Mobile Nodes in Nested Mobile Networks", Proceeding of the International Conference on Computational Science and Its Applications (ICCSA 2006), LNCS 3981, 2006, Part 2, pp 515-524.

22. Koodli, R. Ed., "Fast handoffs for Mobile IPv6". IETF RFC 4068, 2008. 